\title{
IMMEDIATE RADICAL FANG MARK ABLATION MAY ALLOW TREATMENT OF JAPANESE VIPER BITE WITHOUT ANTIVENOM
}

\author{
Fujioka M (1), Oka K (1), Kitamura R (1), Yakabe A (1), Ito M (2)
}

(1) Department of Plastic and Reconstructive Surgery, National Hospital Organization, Nagasaki Medical Center, Nagasaki, Japan; (2) Department of Pathology, National Hospital Organization, Nagasaki Medical Center, Nagasaki, Japan.

\begin{abstract}
Administration of antivenom is currently the standard treatment for snake envenomation. However, it can sometimes cause anaphylactic reactions including urticaria, bronchospasm and hypotension. Furthermore, it may also provoke life-threatening complications, even though the mortality rate is less than $1 \%$. In this study, we present a new treatment - immediate radical fang mark ablation - that was successfully performed on five victims of Japanese viper bites without antivenom use. In these five victims of venomous snakebites, surgical debridement was immediately performed. Two patients received a free-skin graft to resurface their wounds while three of them healed conservatively (i.e. by ointment treatment without surgery). After treatment, all patients could return to work. Immediate radical ablation is a recommended procedure that can reduce the amount of venom in tissues, which consequently decreases inflammatory reactions and reduces the necessity for antivenom.
\end{abstract}

KEY WORDS: ablation, viper bite, antivenom, fang mark, envenomation.

CONFLICTS OF INTEREST: There is no conflict.

\section{CORRESPONDENCE TO:}

MASAKI FUJIOKA, Department of Plastic and Reconstructive Surgery, Nagasaki National Medical Center, 1001-1, Kubara 2, Ohmura City, 856-8562, Japan. Phone: 095752 3121. Fax: 095754 0292. Email: mfujioka@nmc.hosp.go.jp. 


\section{INTRODUCTION}

More than 1,000 cases of Japanese viper (Gloydius b. blomhoffii, Japanese mamushi) bites are believed to occur annually in Japan (1). The venom of these snakes contains a chemically complex matrix of proteins that present enzymatic activities and cause local necrosis. On the other hand, this venom also contains lowmolecular-weight polypeptides that increase capillary membrane permeability, resulting in plasma protein extravasations and red cell fragmentation. This lethal process can occur in many organs, including the lung, kidney, myocardium and central nervous system (2).

The most recent guidelines for first aid against viper envenomation call for avoiding the following measures: ice packs, incision, sucking, tourniquets and hot packs. The recommended therapy for a patient that presents rapid inflammation caused by viper envenomation is the administration of antivenom (3). However, it carries a risk of anaphylactic reaction, so that antivenom usage should be approached with extreme caution.

We successfully treated viper envenomation without antivenom by performing immediate radical fang mark ablation on five patients. This procedure is presented herein as a new first aid measure for victims of venomous snakebites.

\section{CASE REPORTS}

\section{Case 1}

A 72-year-old woman was bitten on her left leg by a Japanese viper and arrived at our unit 50 minutes after the injury. At initial examination, the medial side of the left knee was swollen with an area of ecchymosis and necrotic soft tissue measuring 3.5 $x 2.0 \mathrm{~cm}$ around the fang mark (Figure 1). Surgical debridement of ecchymotic surface, as well as ischemic and necrotic tissue, including the surrounding inflamed skin (total of $8.5 \times 7.0 \mathrm{~cm}$ ) was immediately performed above the fascial layer under local anesthesia.

The wound was left open and wet-to-dry dressings were applied for five days. Severe systemic symptoms, including hypotension, respiratory distress or numbness were not observed, but the serum creatinine kinase (CK) level was remarkably increased $(10,103 \mathrm{IU} / \mathrm{L})$ throughout the subsequent week. The patient received tetanus toxoid (250 IU) and antibiotics (cefazolin, $2 \mathrm{~g} /$ day) intravenously as a prophylaxis against secondary infection. Antivenom was not administered due to the stable general 
condition of the patient, which revealed constant renal function and normal platelet counts, prothrombin time and partial thromboplastin time. Two weeks later, the patient received a split-thickness skin graft to resurface the wound. Six months after the injury, the patient could return to work without any complications (Figure 2).

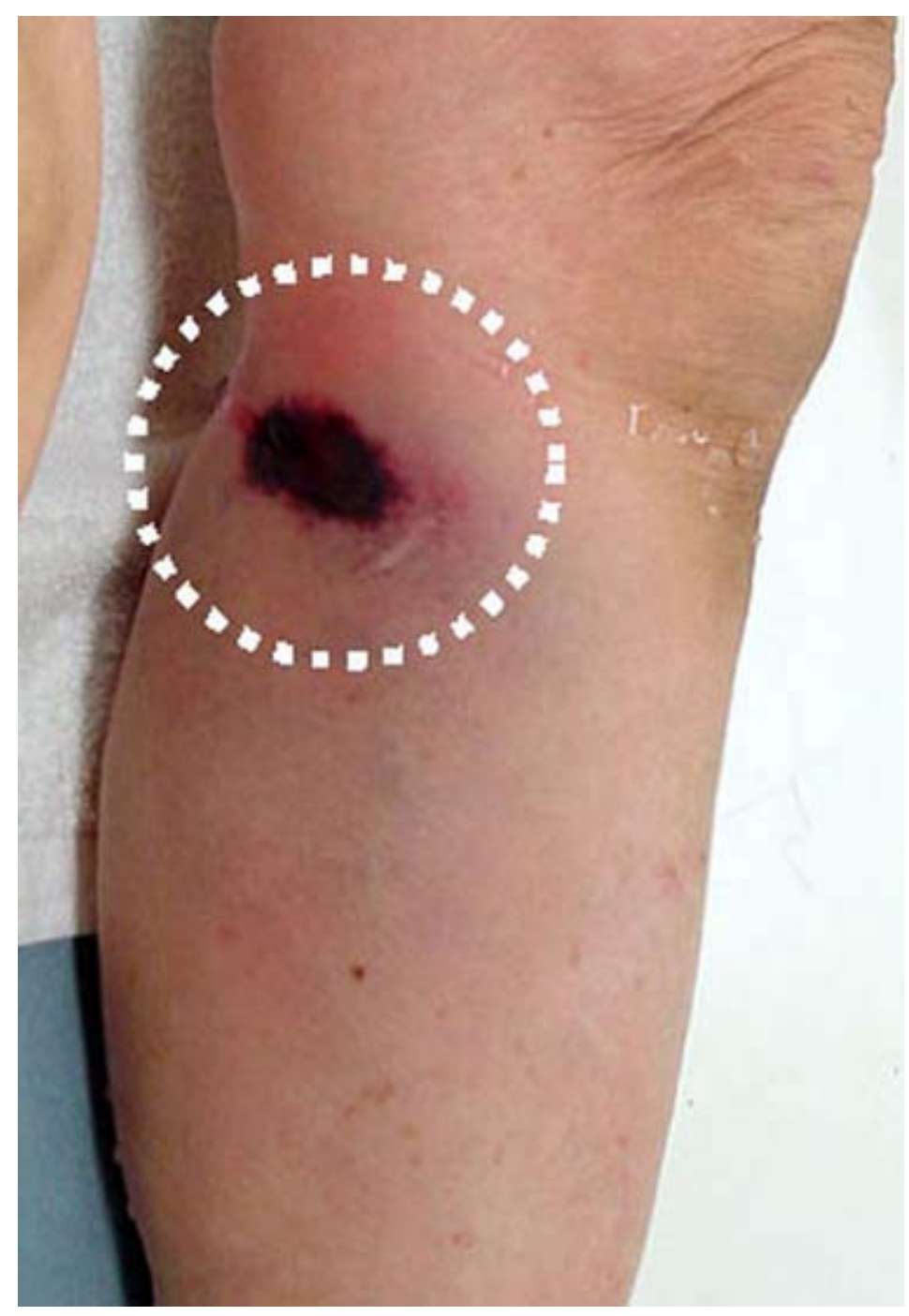

Figure 1. Initial examination showed that the left knee was swollen with an area of soft tissue necrosis measuring $3.5 \times 2.0 \mathrm{~cm}$ around the fang mark. The dotted line indicates the debridement area. 


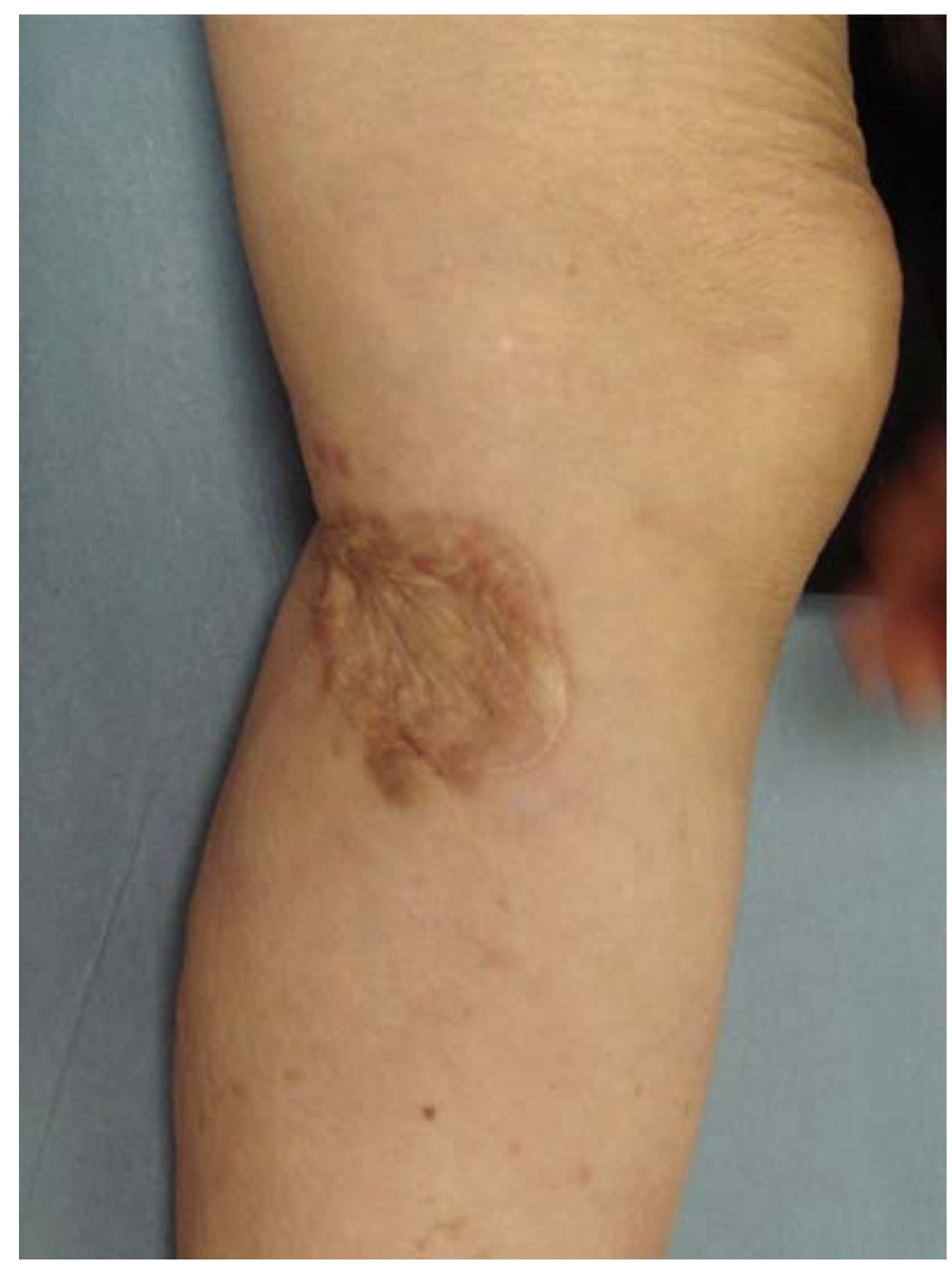

Figure 2. Six months after injury, the patient could return to work.

\section{Case 2}

The left index finger of a 74-year-old man was bitten by a Japanese viper. About 30 minutes after the injury, the victim arrived at our emergency unit where the first examination revealed that the radial side of the finger was swollen with an area of soft tissue necrosis measuring $1.5 \times 0.6 \mathrm{~cm}$ around the fang mark (Figure 3). Immediate ablation was performed on the damaged skin, including the surrounding inflamed surface, covering a total area of $2.0 \times 1.0 \mathrm{~cm}$ (Figure 4). The wound was left open for five days with wet-to-dry dressing. On the next day, severe systemic symptoms were not observed, but CK level was increased (1,248 IU/L). The patient received tetanus toxoid (250 IU) and antibiotics (cefazolin, 2 g/day) intravenously. Antivenom was not administered, since the general condition and laboratory data of 
the patient indicated stability. Histopathological analysis of the fang mark site revealed red cell extravasation, fibrinoid necrosis of vessels and subcutaneous hemorrhagic necrosis (Figure 5). Treatment with ointment was performed, and the wound healed within two months with no sensory or functional impairment (Figure 6).

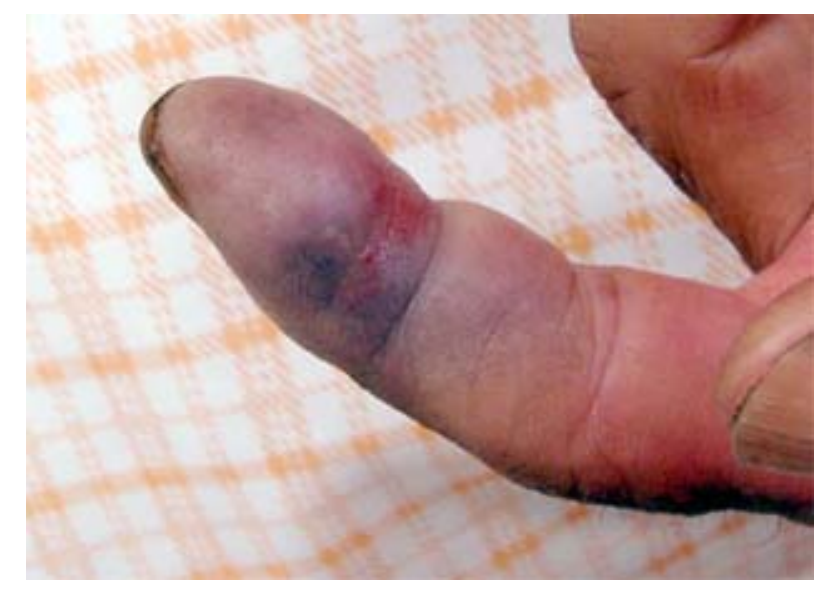

Figure 3. Examination about 40 minutes after injury revealed the swollen radial side of the left index finger with tissue necrosis area around the fang mark of $1.5 \times 0.6 \mathrm{~cm}$.

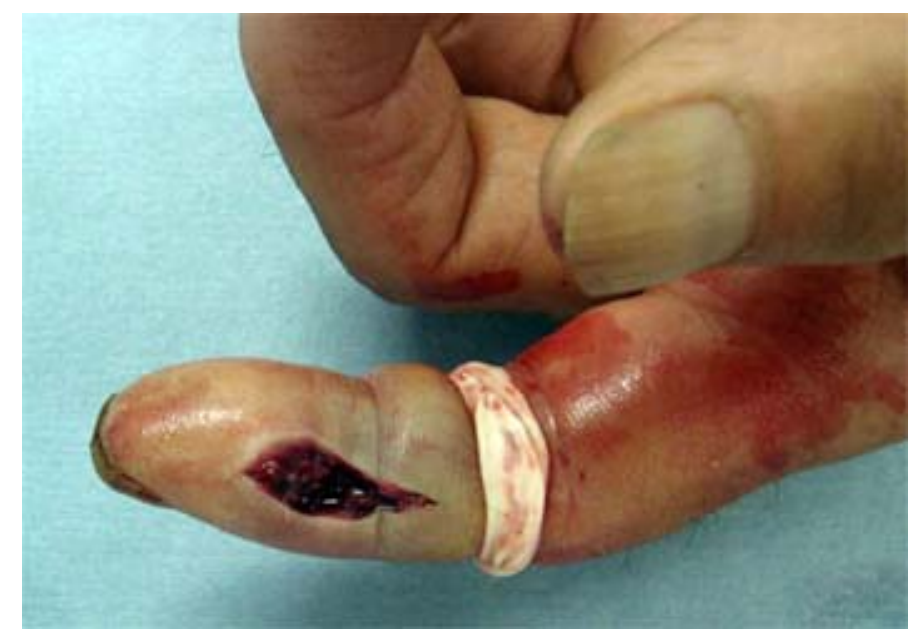

Figure 4. Wound appearance after removal of $2.0 \times 1.0 \mathrm{~cm}$ area of soft tissue. 


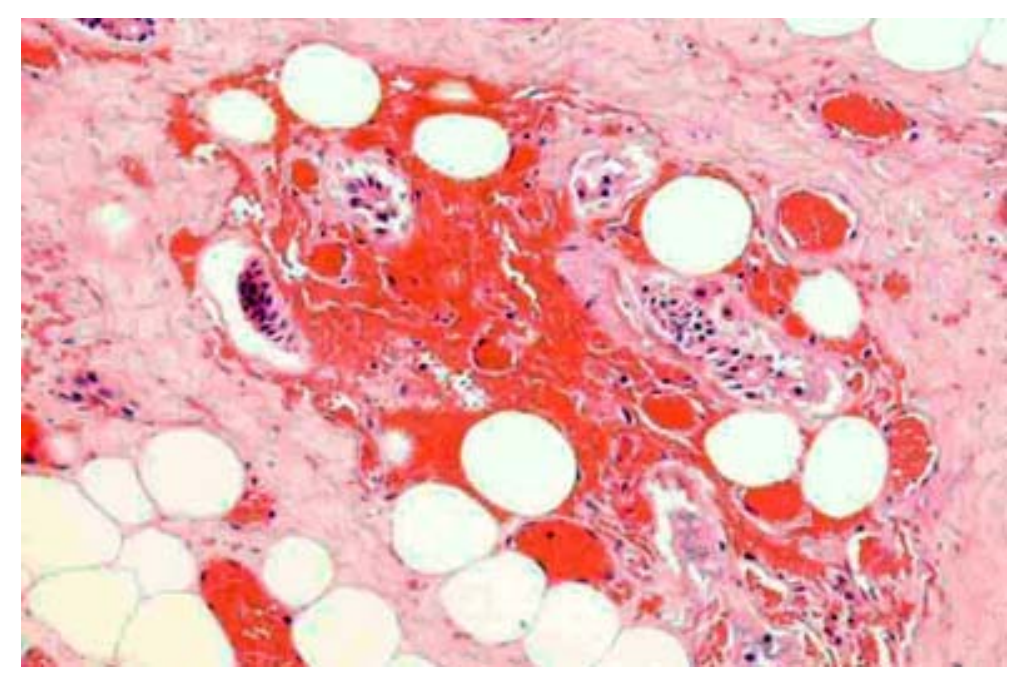

Figure 5. Histopathological findings of the fang mark site immediately after injury. Image shows red cell extravasation, vessel fibrinoid necrosis and subcutaneous hemorrhagic necrosis with neutrophil infiltration.

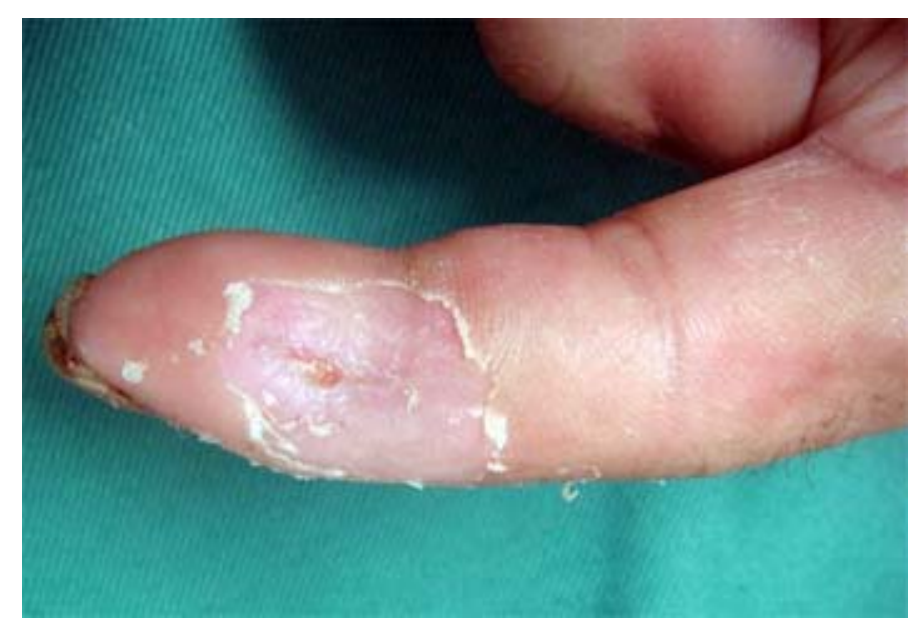

Figure 6. Two months after injury, the wound was completely healed.

\section{Case 3}

A 68-year-old woman was bitten by a Japanese viper on her left ring finger and was transferred to our emergency unit 30 minutes after the accident. The affected finger presented two lesions around fang marks characterized by swelling, ecchymotic area, ischemic soft tissue and measured, respectively, $1.0 \times 0.8 \mathrm{~cm}$ and $0.6 \times 0.5 \mathrm{~cm}$ (Figure 7). The necrotic tissue and inflamed skin areas were immediately ablated $1.2 \times 0.8 \mathrm{~cm}$ in each lesion (Figure 8). The wound was left open and conservative treatment with ointment was employed. Severe systemic symptoms were not observed, but the CK level was increased (11852 IU/L) for the following five days. 
The patient received tetanus toxoid (250 IU) and antibiotics (cefazolin, 2 g/day) intravenously. Antivenom was not administered. The wound healed with slight numbness within 1.5 months (Figure 9).

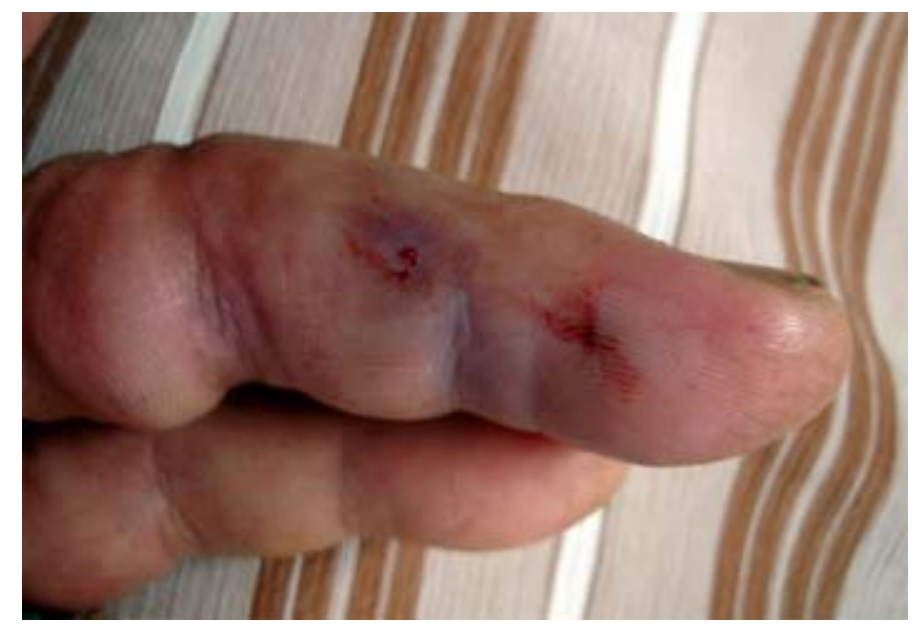

Figure 7. Two lesions on the left ring finger. Swelling and tissue necrosis are visible around fang marks $(1.0 \times 0.8 \mathrm{~cm}$ and $0.6 \times 0.5 \mathrm{~cm})$.

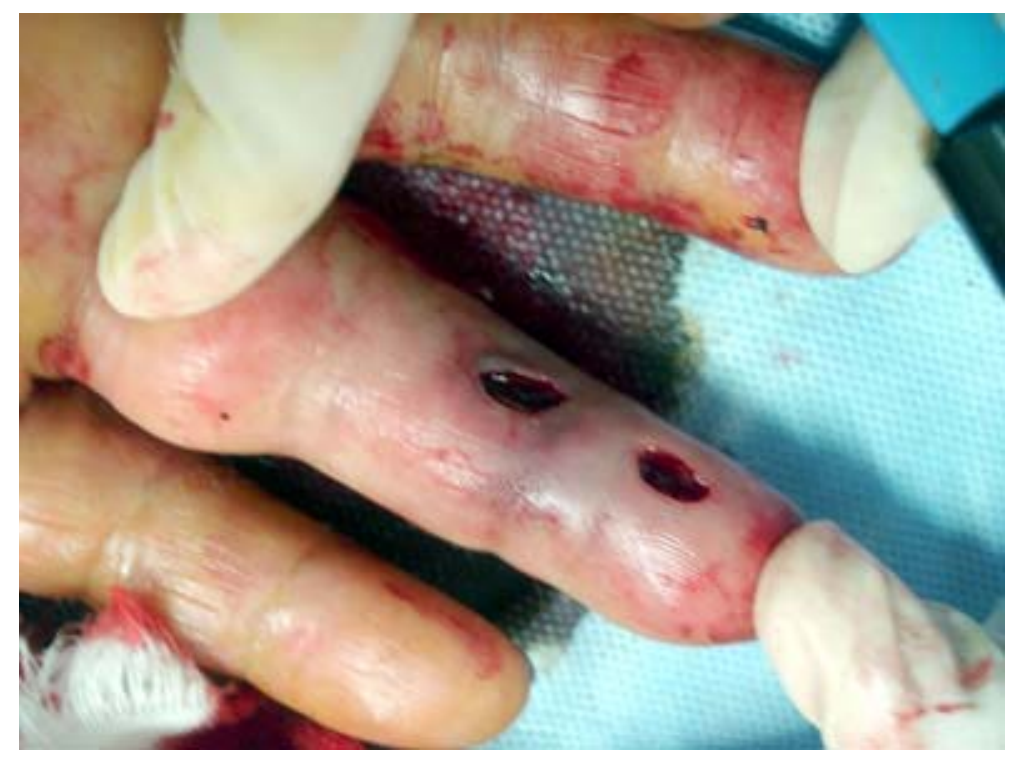

Figure 8. Necrotic soft tissue was immediately ablated. 


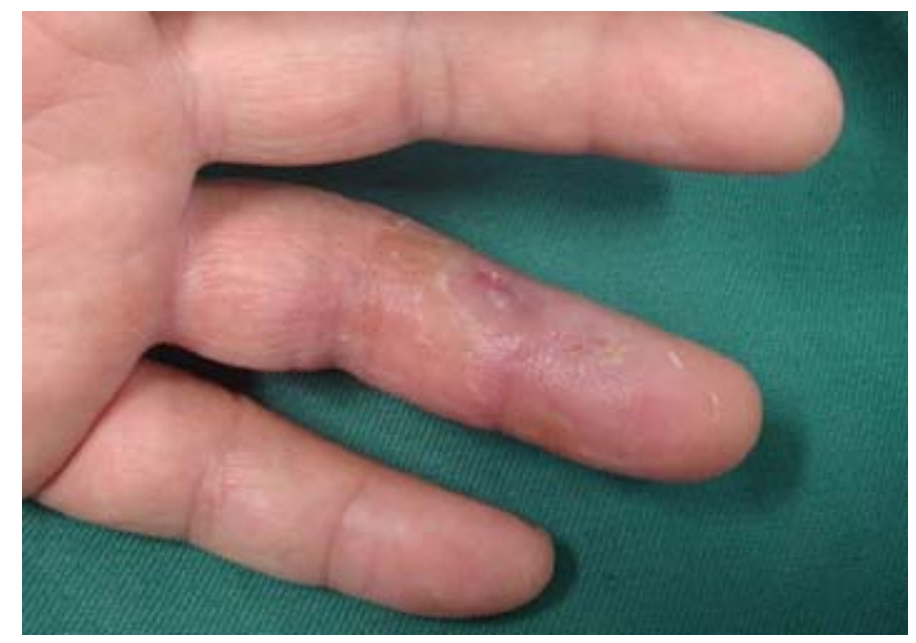

Figure 9. The wound healed with slight sensory disturbance within 1.5 months.

\section{Case 4}

One hour after being bitten by a Japanese viper on the right index finger, a 68-yearold man arrived at our emergency unit. First inspection revealed that the affected finger was swollen with soft-tissue necrosis surrounding the bite $(0.8 \times 0.5 \mathrm{~cm})$. Immediate ablation of the damaged tissue was carried out, including the proximal inflamed skin $(2.5 \times 1.0 \mathrm{~cm})$. The wound was left open and wet-to-dry dressing was applied for five days. The patient did not show systemic symptoms, except for the increased CK level (3988 IU/L) that persisted for a week. The victim received tetanus toxoid (250 IU) and antibiotics (cefazolin, $2 \mathrm{~g} /$ day) intravenously. Since the patient presented satisfactory general condition and stable laboratory tests, antivenom was not administered. To resurface the wound, the patient received a free skin graft three weeks later. Two months after the injury, the lesion was completely healed and the patient could return to work.

\section{Case 5}

A 78-year-old man was bitten on his left index finger by a Japanese viper and was transferred to our emergency unit one hour later. The finger was swollen with ecchymosis as well as ischemic soft tissue around the fang mark $(0.3 \times 0.2 \mathrm{~cm})$. The necrotic tissue and inflamed skin were ablated $(2.5 \times 0.6 \mathrm{~cm})$. The wound was left open and wet-to-dry dressings were applied. Besides a slight increase in the CK level (585 IU/L) for five days, systemic symptoms were not observed. The patient received tetanus toxoid (250 IU) and antibiotics (cefazolin, 2 g/day) intravenously, although antivenom was not administered. Conservative treatment with ointment was 
performed, and the wound healed with no sensory or functional impairment within two months.

\section{DISCUSSION}

The venom of Japanese vipers contains a thrombin-like enzyme $(1,4)$. To treat viper envenomation, the first aid in the 1960s and 1970s included incision, sucking, stifling, and ice packs. However, in 1967, Russel (5) studied 147 patients who had received first aid and 78 who had not, and concluded that there was no evidence that the tratment had made any difference in short-term outcome. Kresanek et al. (6) also evaluated 58 cases of venomous snakebites and concluded that old types of first aid treatments were contradictories, since they can damage vessels and nerves, cause infections and worsen the wounds. For these reasons, old first aid methods are now strongly discouraged and the mainstay of hospital treatment for venomous snakebites is now administration of antivenom (3). Recent estimates suggest that the mortality rate is less than $1 \%$ for patients treated with the antivenom (1).

However, we believe there are some problems with the indiscriminate use of antivenom. The primary problems are anaphylaxis and serum sickness associated with antivenom treatment. Retrospective studies found that acute reactions, such as urticaria, bronchospasm and hypotension, occur in 23 to $56 \%$ of patients during the antivenom infusion (7). Punde (8) investigated 427 patients who received antivenom after envenomation by snakes, and reported that anaphylaxis due to anti-snake venom occurred in $11.7 \%$. Furthermore, Biswas et al. (9) found unexpected severe complications of antivenom-induced myelopathy. Taking into account these studies, the use of antivenom should be prudent or avoided if possible.

Since snakebite severity depends on the amount of venom injected into the victim, if even a small part of it can be removed, patients should present milder symptoms (3). Measures to diminish venom volume into victims' body have been used since ancient times and include cutting and sucking as well as the use of suction devices. However, as previously stated, these first aid treatments are currently of no value and may actually worsen the bite wound $(5,6,10)$.

Immediately after snakebite, envenomation occurs at the bite site causing swelling and skin necrosis around fang marks, which is also evident under histopathological examination $(2,9)$. Histopathological analysis of damaged tissue, including fibrinoid necrosis, vein thrombosis, red blood cell extravasation and subcutaneous tissue 
necrosis, are consistent with the effects of the venom (2). Generally, injected venom diffuses proximally along the subcutaneous flow of lymph, inducing inflammatory reactions in the surrounding soft tissues.

We concluded that acute reactions - such as vascular obstruction and tissue necrosis - and necrotic changes were venom immediate effects, due to the observation of neutrophil infiltration. These types of reactions may be primarily local and delay their spread, so that a high dose of venom might remain close to fang marks for a long time. Therefore, the radical ablation of necrotic tissue and inflamed skin surrounding the bite would help reduce the amount of venom, which consequently decreases inflammatory responses and the necessity of antivenom.

In Case 1, the patient reported severe leg pain while swelling and necrotic tissue were visible on the knee. The bite probably had injected a large volume of venom, since the necrosis around fang marks developed rapidly. In our opinion, the immediate radical ablation of inflamed tissue was effective, even without administration of antivenom.

On the other hand, we have already seen victims of viper bites who had no symptoms or signs of envenomation several hours after injuries. In these dry-bite cases, radical ablation of fang mark should not be performed until local signs of envenomation appear, i.e., ecchymosis, ischemia, swelling, increasing pain and skin necrosis.

Since the aim of this procedure is removal of injected venom, all necrotic soft tissue and inflamed skin must be debrided. The earlier the treatment is performed, the smaller is the area that must be removed. Our five cases suggested that effective venom removal can be expected if ablation is carried out within an hour after bite. Regrettably, if a large soft-tissue defect results from ablation, a surgical resurfacing procedure - like skin graft or local flap transfer - is required, as occurred in Cases 1 and 4.

Although immediate radical ablation can reduce the volume of injected venom, a total removal is impossible. Continuous observation is indispensable after ablation, and if severe systemic symptoms of envenomation occur - including shock intensification, severe local swelling, digestive or neurological symptoms, and continuous clotting abnormalities - antivenom treatment should be indicated with no hesitation. 
Immediate radical ablation is a useful procedure that can reduce the amount of venom in tissue, which, consequently, decreases inflammatory reactions and reduces the necessity of antivenom usage.

\section{REFERENCES}

1. Itoh S, Matsuda S, Tomita Y. Evaluation of 31 viper bite patients. Jap Clin Dermatorogy. 2004;58:104-6.

2. Suga M, Okuda M, Ogasawara $Y$, Yokoyama E, Hamamoto $Y$, Muto M. The histopathology of envenomation by Japanese viper bite. $\mathrm{Br} \mathrm{J}$ Dermatol. 2004;151(1):249-50.

3. Peterson ME. Snake bite: pit vipers. Clin Tech Small Anim Pract. 2006;21(4):17482.

4. Lee JW, Seu JH, Rhee IK, Jin I, Kawamura Y, Park W. Purification and characterization of brevinase, a heterogeneous two-chain fibrinolytic enzyme from the venom of Korean snake, Agkistrodon blomhoffii brevicaudus. Biochem Biophys Res Commun. 1999;260(3):665-70.

5. Russell FE. First-aid for snake venom poisoning. Toxicon. 1967;4(4):285-9.

6. Kresanek J, Plackova S, Caganova B, Klobusicka Z, Batora I, Kresanek I. The new therapy procedures for viper attack. Cent Eur J Public Health. 2004;12: Suppl:S53-4.

7. Dart RC, McNally J. Efficacy, safety, and use of snake antivenoms in the United States. Ann Emerg Med. 2001;37(2):181-8.

8. Punde DP. Management of snake-bite in rural Maharashtra: a 10-year experience. Natl Med J India. 2005;18(2):71-5.

9. Biswas R, Irodi A, Paul A, Ghimere G, Joshi KR, Alurkar VM, Shetty KJ. Antivenom-induced myelopathy in a semipoisonous snakebite. Int $\mathrm{J}$ Clin Pract. 2004;58(6):645-6.

10. Alberts MB, Shalit M, LoGalbo F. Suction for venomous snakebite: a study of "mock venom" extraction in a human model. Ann Emerg Med. 2004;43(2):181-6. 